Check for updates

Cite this: RSC Adv., 2019, 9, 11420

Received 25th December 2018

Accepted 31st March 2019

DOI: 10.1039/c8ra10557j

rsc.li/rsc-advances

\section{Exploring potential biomarkers of coronary heart disease treated by Jing Zhi Guan Xin Pian using high-throughput metabolomics $\dagger$}

\author{
Hui Sun, ${ }^{a}$ Xue-na Li, ${ }^{a}$ Ai-hua Zhang, ${ }^{a}$ Kun-ming Zhang, ${ }^{a}$ Guang-li Yan, ${ }^{a}$ Ying Han, ${ }^{a}$ \\ Fang-fang $\mathrm{Wu}^{\mathrm{b}}$ and $\mathrm{Xi}$-jun Wang (D) *ab
}

Coronary heart disease (CHD) is a relatively complex disease characterized by narrowing of the arterial lumen and reduction of blood flow to the heart. There is no effective early diagnosis and prevention method. Jing Zhi Guan Xin Pian (JZGXP) is a new preparation prepared from the effective extract of Guanxin II. It is made of five components of traditional Chinese medicine and functions by promoting blood circulation and removing blood stasis and is used for the treatment of CHD and angina pectoris. In our study, a CHD rat model was prepared using a high-fat diet combined with intraperitoneal injection of vitamin D3. Clinical biochemical indexes (TG, CHO and HDL-C), histopathology (coronary and myocardial tissue), electrocardiogram and cardiac indexes were used to evaluate the efficacy of JZGXP in the treatment of CHD model rats. UPLC-HDMS-based metabolomics techniques were used to find metabolic profiles, biomarkers and related metabolic pathways in CHD models and to evaluate the effects of JZGXP on them. At the same time, the targets of JZGXP for the treatment of CHD were analyzed. Our study ultimately identified 25 biomarkers associated with CHD models. Further studies found that these 25 biomarkers involved 9 metabolic pathways in the body and found that JZGXP can recall 21 biomarkers in the urine of model rats and these biomarkers involve nine metabolic pathways. Finally, the targets of JZGXP for the treatment of CHD were $\beta$-alanine metabolism and tyrosine metabolism, i.e. amino acids metabolism. This study showed that metabolomics technology is effective for exploring potential biomarkers associated with syndromes or diseases and the therapeutic mechanisms of a traditional Chinese medicine formulation.

\section{Introduction}

CHD is a complex disease with an increasing incidence in developed and developing countries and which is a leading cause of death worldwide. ${ }^{1,2}$ However, there is no reliable method for its early diagnosis and prevention. CHD is characterized by narrowing of the arterial lumen and reduced blood flow to the heart, which seriously affects a patient's living conditions and health, and can lead to disability and premature death. ${ }^{3-6}$ JZGXP is a new dosage form prepared from the effective extract of Guanxin II which is commonly used in the treatment of CHD in clinical practice. It has the effect of anti-

${ }^{a}$ National Chinmedomics Research Center, Sino-America Chinmedomics Technology Collaboration Center, National TCM Key Laboratory of Serum Pharmacochemistry, Laboratory of Metabolomics, Department of Pharmaceutical Analysis, Heilongjiang University of Chinese Medicine, Heping Road 24, Harbin, China. E-mail: xijunwangls@126.com; Fax: +86-451-82110818; Tel: +86-451-82110818

${ }^{b}$ National Engineering Laboratory for the Development of Southwestern Endangered Medicinal Materials, Guangxi Botanical Garden of Medicinal Plant, Nanning, Guangxi, China

† Electronic supplementary information (ESI) available. See DOI: $10.1039 / \mathrm{c} 8 \mathrm{ra} 10557 \mathrm{j}$ myocardial ischemic injury and inhibiting platelet aggregation. ${ }^{7,8}$ JZGXP is made of Chinese herbal medicines such as red peony root, Salvia miltiorrhiza, chuanxiong, safflower and Lignum Dalbergiae Odoriferae. They have the function of promoting blood circulation and removing blood stasis and are used for chest sputum caused by internal hemorrhage. Symptoms include chest tightness and stinging in the precordial area. JZGXP can be used to treat CHD and angina pectoris, etc. ${ }^{9}$ Index components included in the JZGXP include: salvianolic acid $\mathrm{B}$, paeoniflorin, ferulic acid, tanshinone IIA, safflower yellow A, dihydrotanshinone I, tanshinone I, Danshensu, cryptotanshinone, rosmarinic acid, and the like. Presently, comprehensive studies of the chemical compositions of five traditional Chinese medicines in JZGXP have been made, but analysis of their chemical components is still at the research level with characteristic peaks. Biomarkers that exert a holistic therapeutic effect have not been elucidated. Therefore, based on the CHD model, this study applied the traditional Chinese medicine prescription metabolomics strategy and found the biomarkers and therapeutic mechanisms of JZGXP in the treatment of CHD. ${ }^{\mathbf{1 0 - 1 2}}$ Application of metabolomics methods to evaluate the efficacy and safety of clinical medicine, research on 
the mechanism of action of drugs, especially traditional Chinese medicine, is of great significance. ${ }^{13,14}$

\section{Experimental methods}

\subsection{Chemicals and reagents}

A HO kit (Zhongsheng Beikong Biotechnology Co., Ltd.), TG kit (Zhongsheng Beikong Biotechnology Co., Ltd.), HDL-C kit (Zhongsheng Beikong Biotechnology Co., Ltd.), vitamin D3 injection (1 mL: $7.5 \mathrm{mg}$, National Pharmaceutical Standard H31021404, Shanghai General Pharmaceutical Co., Ltd., China), distilled water (Guangzhou Watsons Food \& Beverage Co., Ltd.), pentobarbital sodium powder (Shanghai Chemical Reagent Purchasing and Supply Station), sodium chloride injection (Harbin Sanlian Pharmaceutical Co., Ltd.), JZGXP (Zhongguo Zhunzi Z23021172, Wusulijiang Pharmaceutical Co., Ltd. Harbin Branch; Heilongjiang), aspirin enteric-coated tablets (National Medicine Zhunzi H20065051, Shenyang Aojina Pharmaceutical Co., Ltd., China), distilled water (Guangzhou Watsons Food \& Beverage Co., Ltd.), acetonitrile (chromatographic grade, Merck, Germany), methanol (chromatographic grade, Fisher, USA), formic acid (chromatographic grade, Tianjin Komi Chemical Reagent Co., Ltd.), leucine enkephalin (SIGMA), other reagents and drugs also meet the experimental requirements.

\subsection{Animal handling and ethical statement}

Male Sprague-Dawley rats were used in the experiment. After successful modeling, the rats were divided into groups and then corresponding drugs were administered. Specific modeling methods, grouping, dosage, etc., are provided in the ESI. $\dagger$ Experimental procedures were approved by the Animal Care and Ethics Committee at Heilongjiang University of Chinese Medicine and all experiments were performed in accordance to the declaration of Helsinki.

\subsection{Sample collection and preparation}

2.3.1 Collection and preparation of serum samples. On the 28th day after administration of the drug, the serum of the blank group, model group, positive group, and the different doses of the JZGXP administration group were collected. Before collecting blood, the rats were fasted for 12 hours and were free to drink water. Anesthesia was performed by intraperitoneal injection of $0.2 \mathrm{~mL}$ of sodium pentobarbital $(0.3 \%)$ per $100 \mathrm{~g}$ of rat body weight. After anesthesia, blood was collected from the abdominal aorta. The collected blood was allowed to stand at room temperature for $30 \mathrm{~min}$, centrifuged $\left(20^{\circ} \mathrm{C}, 4000 \mathrm{rpm}\right)$ for $10 \mathrm{~min}$, and the supernatant was centrifuged for 5 min under the same centrifugation conditions. Then the supernatant was dispensed to a centrifuge tube and stored at $-80^{\circ} \mathrm{C}$.

2.3.2 Collection and preparation of coronary artery and myocardial tissue. Because coronary tissue is small and difficult to peel off, this study took the whole heart directly and studied the coronary and myocardial tissues of the rats in the blank group, model group, positive group, and the different doses of JZGXP drug delivery group. After opening a rat's thoracic cavity, it was first perfused with normal saline, residual blood in the blood vessels was removed and followed by perfusion with $10 \%$ neutral formalin solution to cause an internal fixation. The hearts of each group of rats were removed and placed in a $10 \%$ neutral formalin solution for $24 \mathrm{~h}$ for HE staining.

2.3.3 Collection and preparation of urine samples. Urine samples from the blank group and model group rats were collected at the 7th, 14th, 21st, 28th, 35th, and 42nd day time points of modeling. Urine also was collected from each group at four time points of administration which were on the 7th, 14th, 21st, and 28th days of administration. The urine was centrifuged at $13000 \mathrm{rpm}$ for $10 \mathrm{~min}$ at $4{ }^{\circ} \mathrm{C}$. The supernatant was taken, diluted 5 times, filtered through a $0.22 \mu \mathrm{m}$ microporous membrane, and the filtrate was transferred to a sample cup for analysis by UPLC-HDMS.

\subsection{Evaluation of therapeutic effects}

2.4.1 Determination of electrocardiograms. After 28 days of continuous treatment, electrocardiograms of rats in the blank group, model group, positive group and drug-administered group were collected. Before the collection, the rats in each group were fasted for 12 hours and were free to drink water. After anesthesia with $3 \%$ sodium pentobarbital, electrocardiograms of the blank group and model group were collected using the MD3000 biosignal acquisition and processing system.

2.4.2 Determination of CHO, TG and HDL-C in serum. After 28 consecutive days of treatment, serum samples from the blank group, model group, positive group, and each dose group were measured. The contents of CHO, TG and HDL-C in the serum of each group of rats were determined according to the instructions of the CHO, TG and HDL-C kits.

2.4.3 Determination of cardiac index. After 28 consecutive days of treatment, the body weights of each group of rats was measured. Before weighing, the rats were fasted for 12 hours and allowed to drink freely. After anesthetizing the rats with $3 \%$ sodium pentobarbital, their blood was collected, then the thoracic cavity was opened, the hearts of the rats were taken out and the weight of each was measured.

2.4.4 Histopathological evaluation. After 28 days of continuous treatment, HE staining was performed on the coronary and myocardial tissues of the rats in the blank group, model group, and the positive group. The specific process was as follows: (1) Remove the fixed heart, cut it into $1 \times 0.6 \times$ $0.2 \mathrm{~cm}$ tissue blocks with a knife, and fix in $10 \%$ neutral formalin solution for 24 hours. (2) Use $80 \%, 95 \%, 100 \%$ gradient ethanol elution. (3) Make tissue xylene transparent. (4) Embed in paraffin. (5) Section the heart paraffin; thickness 5 $\mu \mathrm{m}$, then HE stain, resin seal and determine pathological changes under a light microscope.

\subsection{Metabolic profile analysis}

Urine samples of the blank group and model group were collected at six time points: 7 days, 14 days, 21 days, 28 days, 35 days, and 42 days. However, urine of the rats in the administration group was collected at four time points: on day 7, day 14, day 21 and day 28 of dosing, respectively. The liquid-quality 
data collected by the UPLC-HDMS was imported into Waters Progenesis QI software via its Import data module. The imported urine sample data was classified in the same group by the Between-subject Design module. Change parameters were set under the Peak Picking peak program to further extract peaks in the urine sample. Unsupervised PCA was used to obtain the corresponding urine metabolism profiles at the corresponding time points in the blank group and model group and after the administrations.

\subsection{Analysis of biomarkers and metabolic pathways}

2.6.1 Biomarker identification. Urine analysis conditions: (1) mass spectrometry conditions in positive ion mode: capillary voltage $2.6 \mathrm{kV}$, sample cone voltage $30 \mathrm{~V}$, extraction cone voltage $3.0 \mathrm{~V}$, ion source temperature $110{ }^{\circ} \mathrm{C}$, desolvation gas temperature $300{ }^{\circ} \mathrm{C}$, desolvation gas flow $600 \mathrm{~L} \mathrm{~h}^{-1}$. Locking mass solution: online real-time calibration using the Waters Lockspray calibration system, leucine-enkephalin $\left([\mathrm{M}+\mathrm{H}]^{+}=\right.$ 556.2771 ), solution concentration $0.2 \mathrm{ng} \mu \mathrm{L}^{-1}$; flow rate 0.1 $\mathrm{mL} \min ^{-1}$. The quality scan range was 50-1500 Da, scan time was $0.2 \mathrm{~s}$, and the data acquisition mode was centroid. MS conditions in the negative ion mode: capillary voltage $2.6 \mathrm{kV}$, sample cone voltage $35 \mathrm{~V}$, extraction cone voltage $3.5 \mathrm{~V}$, ion source temperature $110{ }^{\circ} \mathrm{C}$, desolvation gas temperature $350{ }^{\circ} \mathrm{C}$, de-solvent gas flow $600 \mathrm{~L} \mathrm{~h}^{-1}$. Locking mass solution: online real-time calibration using the Waters Lockspray calibration system, leucine-enkephalin $\left([\mathrm{MH}]^{-}=554.2620\right)$, solution concentration $0.2 \mathrm{ng} \mu \mathrm{L}^{-1}$; flow rate $0.1 \mathrm{~mL} \mathrm{~min}^{-1}$; mass scan range 50-1500 Da, scan time was $0.2 \mathrm{~s}$; data acquisition mode was centroid. (2) Chromatographic conditions: the column used in this study was a Waters ACQUITY UPLC® HSS T3 $(2.1 \times 100$ $\mathrm{mm}, 1.8 \mu \mathrm{m})$. The elution gradient of urine samples in the positive ion mode is shown in Table $2-1 . \dagger$ In the negative ion mode, the elution gradient of urine samples is shown in Table $2-2 . \dagger$ In both positive and negative ion modes: mobile phase A: $0.1 \%$ formic acid/acetonitrile, mobile phase B: $0.1 \%$ formic acid/water; column temperature was $40^{\circ} \mathrm{C}$. The flow rate was 0.4 $\mathrm{mL} \min ^{-1}$ and the injection volume was $4 \mu \mathrm{L}$.

Corresponding urine metabolism profiles of the blank group and model group were based on PCA analysis and the 42nd day of modeling was selected as the key day for urinary metabolomics analysis. First, PCA analysis was performed on the urine samples of the blank group and model group rats on the 42nd day of modeling, and EZinfo2.0 software ${ }^{47}$ was further used for OPLS-DA analysis to obtain the corresponding Score plot. Further analysis was carried out to obtain S-Plot and VIPPlot maps that directly reflected the contribution rates between groups. Finally, ions with a VIP value greater than 1.5 and $P$ less than 0.05 after statistical analysis software $T$ test were selected as biomarker candidate ions. The identification module was identified by "Identify Compounds" in the Progenesis QI software. Candidate ions were searched in the Human Metabolome Database to obtain possible compound information, and the MOL files of the corresponding ions were downloaded. Further, the secondary ion informations were input into the Massfragment module nested in Masslynx 4.1 software. According to the possibility of chemical structure cleavage and the cleavage of biological mass spectrometry, assignments of candidate ions were determined as biomarkers. The method for analyzing biomarkers in each group after administration was the same as above.

2.6.2 Metabolic pathway analysis. MetPA (Metabolomics Pathway Analysis) is a network tool designed to analyze and visualize metabolic pathways with a biological background in metabolomics information. A variety of advanced path analytical programs were combined to analyze the topological characteristics of metabolic pathways of metabolites, and to find those metabolic pathways with the strongest correlation of the analyzed metabolites. Biomarkers in the obtained urine samples were imported into the Pathway Analysis module on the Metaboanalyst website to analyze the metabolic pathways involved.

\section{Results}

\subsection{Therapeutic effects}

3.1.1 Electrocardiogram. After 28 days of treatment, electrocardiograms of the rats in the blank group, model group, positive group and the administration group were collected. The electrocardiogram results showed that the model group, positive group and the rats in each administration group showed negative myocardial ischemia, indicating that the JZGXP and aspirin treatment or natural recovery, myocardial deficiency and myocardial ischemia status of the rats were alleviated. The blood condition disappeared. The electrocardiogram results are shown in Fig. $\mathrm{S} 1 . \dagger$

3.1.2 Serum TG, CHO, HDL-C content. After 28 days of treatment, the contents of TG, CHO and HDL-C in the serum of the blank group, model group, positive group and the respective dose-administered groups were determined. The detailed results are shown in Fig. $\mathrm{S} 2 \uparrow$ and showed that compared with the model group, serum levels of TG and $\mathrm{CHO}$ in the rats treated with different doses of JZGXP decreased, and there was a significant difference. The serum HDL-C content in rats increased. There was also a marked difference between the medium dose group and the high dose group. These results showed that JZGXP could improve the blood lipid level of rats, and the high-dose group of JZGXP had the best effect. In contrast with the blank group, the serum levels of TG and CHO in the model group increased, and the content of HDL-C decreased, indicating that the model group was still in CHD after 4 weeks of natural recovery. In addition, compared with the model group, the serum levels of TG and $\mathrm{CHO}$ in the positive group decreased, and there was no significant difference. The serum HDL-C content in the rats also increased with no significant differences.

3.1.3 Cardiac index. The cardiac index can reflect the extent of the therapeutic effect of JZGXP on CHD model rats and detailed results are shown in Fig. S3. $\dagger$ It can be seen from the figure that after 28 days of continuous treatment, in contrast with the model group, the cardiac index of the rats with the different doses of JZGXP increased, and the JZGXPs were administered at high doses. There was a very significant 
difference $(P<0.01)$ which indicated that JZGXP improved the heart index of rats. In contrast with the model group, the cardiac index of the rats in the positive group also increased, and there was a marked difference $(P<0.05)$ which indicated that aspirin also changed the heart index of rats to a certain extent.

3.1.4 Histopathology. After 28 consecutive days of treatment, HE staining was performed on the coronary and myocardial tissues of the rats in the blank group, model group, positive group, and the different doses of the JZGXP. The results are shown in Fig. S4. $\dagger$ Results of HE staining in the coronary artery and myocardial tissue showed that the endothelial cells in the blank group were intact, they were arranged neatly, and the myocardial fiber bundles also were evenly arranged. However, endothelial cells in the model group were irregularly arranged, shedding occurred, and a small amount of lipid cells were deposited. At the same time, there was a certain degree of myocardial fibrosis; in contrast with the model group, lipid deposition in the positive group was reduced, the arrangement of endothelial cells was regularized, and the degree of myocardial fibrosis was reduced, indicating that aspirin is a model of CHD. It can play a certain degree of therapeutic effects; in contrast with the model group, the deposition of lipid cells in the rats with different doses of JZGXP was significantly reduced, deposition of calcium salts disappeared, and the degree of myocardial fibrosis was significantly improved. Thus, the highdose group of JZGXP significantly improved the atherosclerosis of rats.

\subsection{Metabolic profile of CHD rats}

PCA was used to analyze the ESI-MS data of the $7 \mathrm{~d}, 14 \mathrm{~d}, 21 \mathrm{~d}$, 28 d, 35 d, 42 d blank and model group urine samples, and score plots from six times were obtained respectively, as shown in Fig. 1. It can be seen from the figure that from the 7th to the 42nd day, urine samples of the blank group and model group can be clearly separated, indicating that the endogenous components in the urine of the model group were disturbed since the 7 th day of modeling until the 42 nd day.

\subsection{Biomarkers and metabolic pathway analysis}

The BPI chromatograms obtained from the urine sample in positive and negative ion modes are shown in Fig. 2 . In order to avoid omission of important biomarkers, the 42 nd day of modeling, which has significant differences in various indicators, was selected as the key day for model replication, and biomarkers of the rat model of CHD were found and identified. First, a non-supervised PCA analysis of urine samples from the key day blank group and model group rats was carried out to obtain the corresponding Score plot. The score map is shown in Fig. 3. Secondly, a supervised OPLS-DA analysis was performed to obtain its corresponding Score plot. The score chart is shown in Fig. 4, and the S-Plot and VIP-Plot maps which reflect differences in contribution rates between the two groups, are shown in Fig. 5. It can be seen from the PCA score chart that urine samples of the model group and blank group can be clearly distinguished on the 42nd day of modeling, indicating the endogenous nature of the model group rats produced significant disturbances after treatment with a high fat diet combined with intraperitoneal injection of vitamin D3 metabolites.

Chemical structures of key metabolites were confirmed by the identification method mentioned above. Finally, 25 endogenous biomarkers related to the coronary heart disease model were identified, in which 4,6-dihydroxyquinoline and 3methyldioxane were detected in both positive and negative ion modes. In the positive ion mode, 13 biomarkers were identified, and in the negative ion mode, 14 biomarkers were identified. At the 42nd day of modeling, 15 biomarkers in urine of the CHD model group showed an upward trend compared with the blank group. The detailed results are shown in Fig. 6 .

Based on the above methods, 25 biomarkers were ultimately involved in the 9 metabolic pathways. The results are shown in Fig. 7. The 9 metabolic pathways can be further classified into 5 primary metabolic pathways: an amino acid metabolic pathway, carbohydrate metabolism, nucleotide metabolism, metabolism of cofactors and vitamins and metabolism of other amino acids. Among them, the amino acid metabolism includes phenylalanine metabolism, tryptophan and tyrosine metabolism while the carbohydrate metabolism includes starch and sucrose metabolism, propionate metabolism and ascorbate metabolism. The nucleotide metabolic pathway includes pyrimidine metabolism while the vitamins and coenzymes factor metabolic pathways includes pantothenate and coenzyme A biosynthesis; another amino acid metabolism includes $\beta$-alanine metabolism.

As can be seen from Fig. 7, CHD can mainly cause $\beta$-alanine metabolism disorders and tyrosine metabolism disorders in the body. The biomarkers $\beta$-alanine and homovanillic acid sulfate associated with CHD screened in this experiment are important intermediate metabolites of $\beta$-alanine metabolism and tyrosine metabolism, respectively. In contrast with the blank group, $\beta$ alanine in the model group was increased visibly $(P<0.01)$, and the high vanillic acid sulfate content also was increased visibly $(P<0.05)$ due to disorder of the amino acid metabolism in the body from CHD.

\subsection{Effect of JZGXP on metabolic profiles}

PCA was used to analyze the ESI-MS data of urine samples 28 days after administration to obtain a score plot, as shown in Fig. 8. It can be seen from the figure that urine samples of the low, medium and high dose groups of the blank group, model group, positive group and the JZGXP can be clearly separated, indicating that JZGXP can change the metabolism of rats, and the high-dose group of JZGXP was the best.

\subsection{Effects of JZGXP on biomarkers and metabolic pathways}

UPLC-HDMS data was collected into rat urine data and processed using Waters Progenesis ${ }^{\circledR}$ QI software. After 28 days of treatment, by analyzing the changes in the urine content of the biomarkers identified in the successful model of CHD in each group of rats, it was found that aspirin and different doses of JZGXP made most of the biomarkers in the urine turn back in 

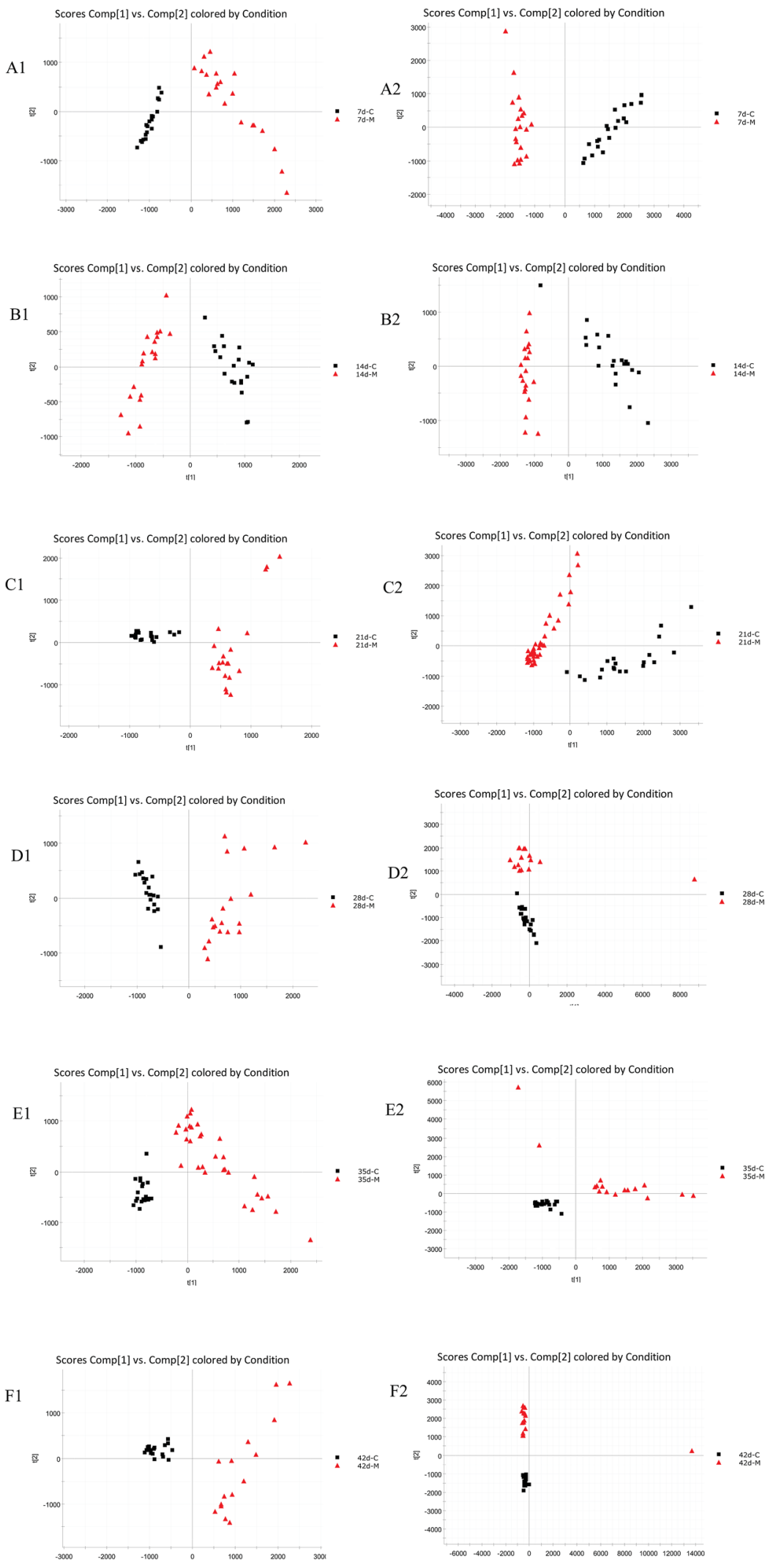

Fig. 1 Urine metabolic profiling of two rat groups on modeling $7 d, 14 d, 21 d, 28 d, 35 d$, and $42 d$ based on PCA analysis. (A1-F1) each point of time in positive ion mode; (A2-F2) each point of time in negative ion mode. C: control group; M: model group. 


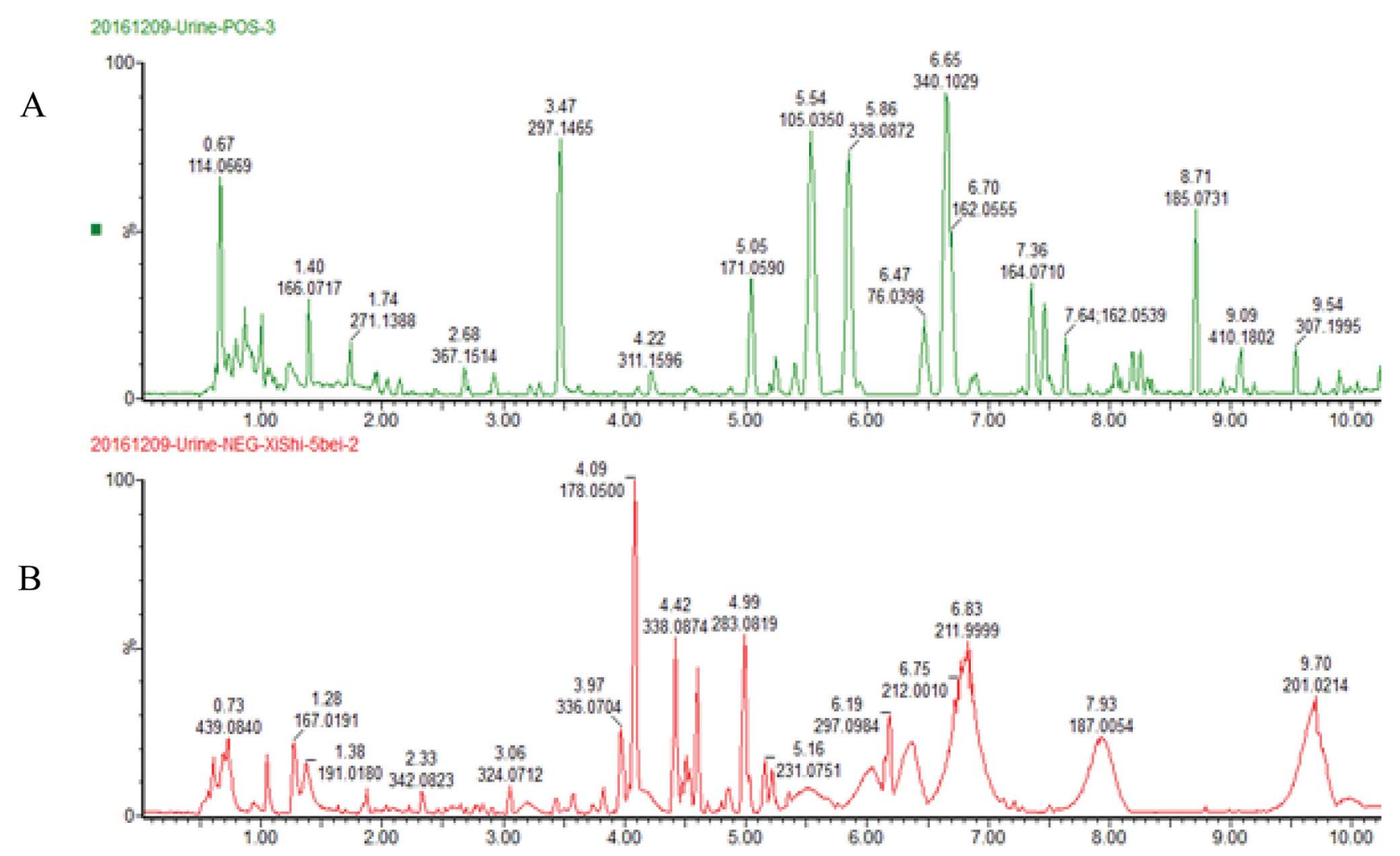

Fig. 2 UPLC-HDMS chromatograms of urine samples in positive and negative ion mode. (A) positive ion mode; (B) negative ion mode.

the direction of the blank group. The content was adjusted back toward the blank group and detailed results are shown in Fig. 9. Among the 25 urine biomarkers associated with the CHD model, the JZGXP recalled 21 biomarkers. From a statistical point of view, the callback of 8 biomarkers in the JZGXP highdose group was statistically significant. In contrast with the model group, there were marked differences between the 6 biomarkers, and 2 biomarkers had extremely significant differences. The callback of 10 biomarkers in the JZGXP middledose group also was statistically significant. In contrast with the model group, 9 biomarkers had marked differences, 1 biomarker had extremely significant differences, and with 5 biomarkers in the low-dose group of JZGXP the callbacks were statistically significant, with 5 biomarkers having significant differences compared to the model group. Then, 21 biomarkers of the above callbacks were introduced into the MetPA website to analyze related metabolic pathways. This study found that the main metabolic pathways involved were amino acid metabolic pathways, carbohydrate metabolic pathways, nucleotide metabolic pathways, vitamin and coenzyme metabolic pathways, and another amino acid metabolic pathway. Among them, the amino acid metabolism includes phenylalanine
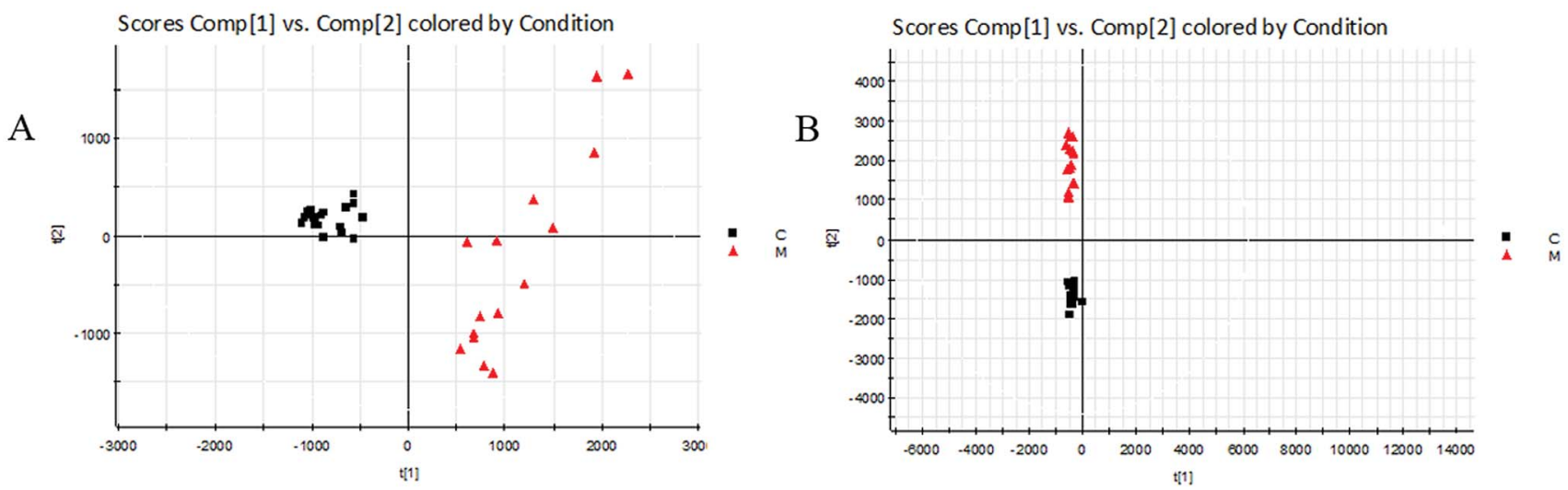

Fig. 3 Score plots of urine metabolic profiling of two rat groups after modeling 42 days and analyzed by PCA. C: control group; M: model group; (A) positive ion mode; (B) negative ion mode. 

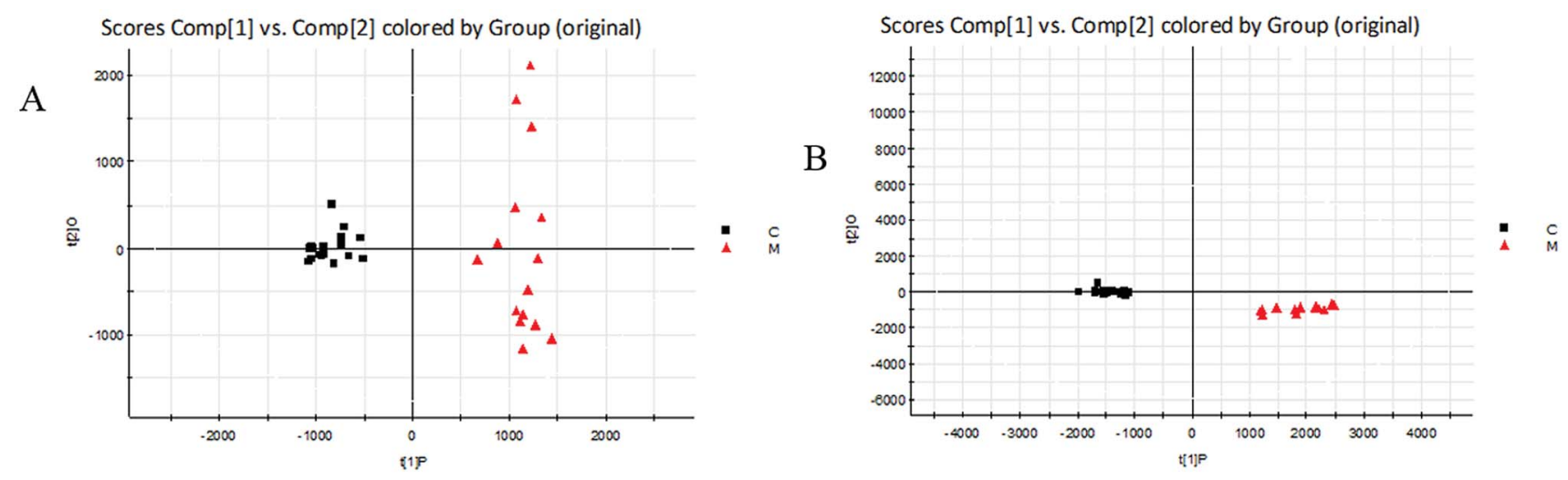

Fig. 4 Score plots of urine metabolic profiling of two rat groups after modeling 42 days and analyzed by OPLS-DA. C: control group; M: model group; (A) positive ion mode; (B) negative ion mode.

metabolism, tryptophan metabolism and tyrosine while the carbohydrate metabolism includes starch and sucrose metabolism, propionate metabolism and ascorbate metabolism. The nucleotide metabolic pathway includes pyrimidine metabolism while vitamins and coenzymes factor metabolism pathways include pantothenate and coenzyme A biosynthesis. Other amino acid metabolisms include beta-alanine metabolism. So, in total 25 biomarkers were involved in the 9 metabolic pathways.

\subsection{Targets analysis}

CHD can mainly cause disorders of amino acid metabolism in the body, including $\beta$-alanine metabolism disorder and tyrosine metabolism disorder. Biomarkers directly related to $\beta$-alanine metabolism and tyrosine metabolism are $\beta$-alanine and high vanillic acid sulfate. After treatment with JZGXP in the model group, the levels of $\beta$-alanine and homovanillic acid sulfate in the body can be adjusted back to the normal group. Through
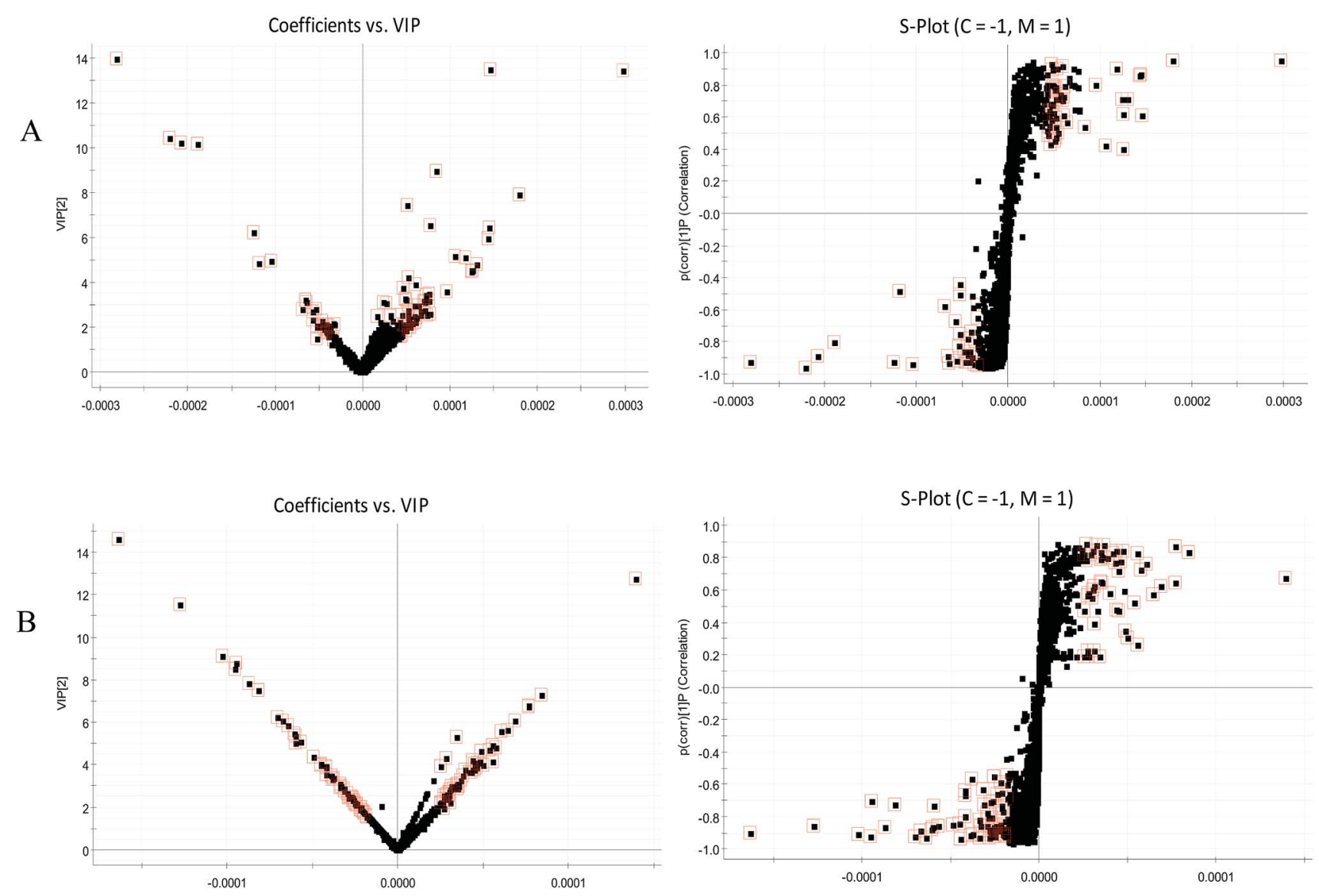

Fig. 5 VIP-plots and S-plots of urine metabolic profiling of two rat groups after modeling 42 days and analyzed by OPLS-DA. (A) positive ion mode; (B) negative ion mode. 

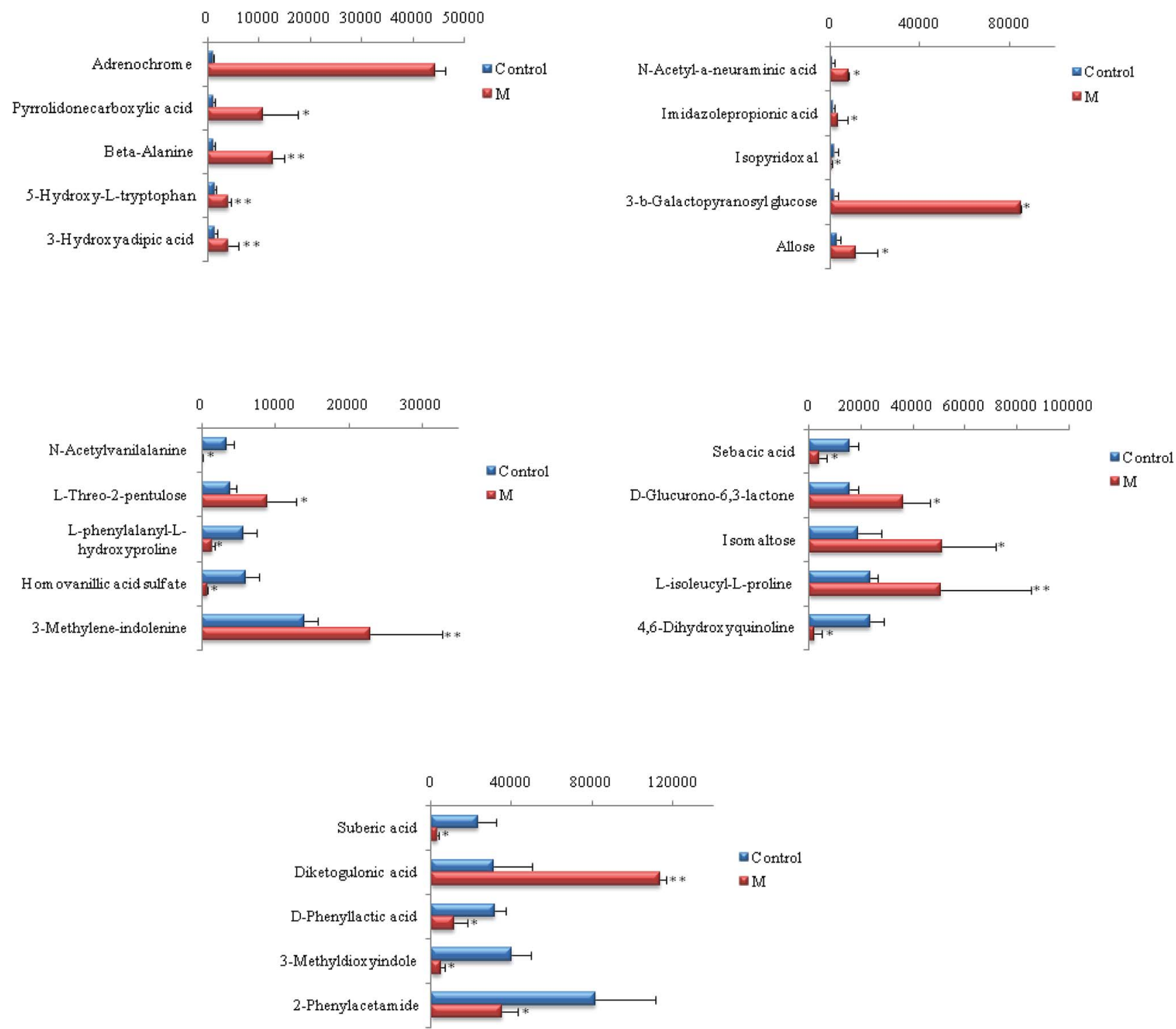

Fig. 6 Content changes of biomarkers after modeling 42 day old rat urine. ( $* P<0.05, * * P<0.01$ compared to the control group.) Control: control group; M: model group.

the above and Fig. 7, etc., we can hypothesize that the targets of JZGXP in the treatment of CHD may be $\beta$-alanine metabolism and tyrosine metabolism.

\section{Discussion}

JZGXP is made from Chinese herbal medicines such as red peony root, Salvia miltiorrhiza, chuanxiong, safflower and Lignum Dalbergiae Odoriferae. Salvia miltiorrhiza is the dried root and rhizome of the Salvia miltiorrhiza Bge., which can relieve pain, regulate blood circulation and nourish the heart and calm the nerves. It mainly includes diterpenoids and phenolic acids. The diterpenoids are mainly tanshinone II, tanshinone I, isotanshinone I, II, etc. while the phenolic acids mainly include salvianolic acid A and salvianolic acid. This plant has functions of regulating cardiovascular and antithrombotic symptoms. Red peony root is the dry root of Paeonia lactiflora Pall. or Paeonia veitchii Lynch. It can calm the liver and relieve pain, nourish the blood, regulate the yin and relieve sweat. This plant mainly contains monoterpenes and their glycosides, mainly paeoniflorin, and contains a small amount of hydroxy peonidin. It can treat myocardial ischemia, and a study found that red peony root and safflower also can play a role in promoting blood circulation. Chuanxiong is derived from the dried rhizome of the umbelliferous plant Ligusticum chuanxiong Hort. This plant activates blood and relieves pain. It mainly contains three components consisting of volatile oils, alkaloids and phenolic acids. Volatile oils include decidual lactones, butenyl azlactone, alkaloids such as ligustrazine, uracil, etc., plus phenolic acids such as chuanxiong, ferulic acid, and similar compounds. It has anti-atherosclerosis and anti-ischemic effects. Safflower is a dry tubular flower of the 


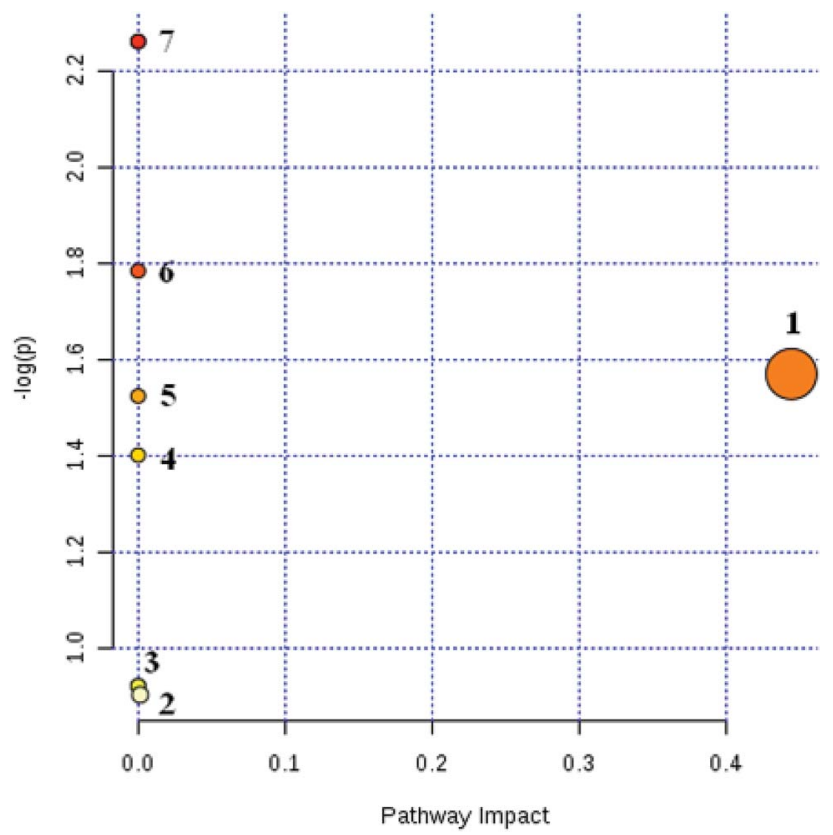

Fig. 7 Pathway analysis with MetPA of CHD model rats. (1) $\beta$-Alanine metabolism; (2) tyrosine metabolism; (3) tryptophan metabolism; (4) starch and sucrose metabolism; (5) propanoate metabolism; (6) pantothenate and CoA biosynthesis; (7) phenylalanine metabolism.

genus Carthamus tinctorius L. This plant can promote blood circulation and relieve pain. The chemical composition of a safflower plant is relatively complex, mainly containing dihydroflavones, lignans, polysaccharides, volatile oils, fatty oils and organic acids. The flavonoids are mainly safflower yellow pigments, including hydroxy safflower yellow A, safflower yellow $\mathrm{B}$, safflower yellow $\mathrm{C}$ and similar compounds. It has an anticoagulant, antithrombotic effect and hypolipidemic effect. Lignum Dalbergiae Odoriferae include the legumes Dalbergia odorifera T. Chen trunk and root dry heartwood. This plant has the effects of relieving pain, promoting blood circulation and stopping bleeding. The main chemical components in the Lignum Dalbergiae Odoriferae are volatile oils and flavonoids. The volatile oils mainly include nerolidol, 2,4-dimethyl-2,4heptadienal, etc. while the flavonoids mainly include isoflavones, chalcones and dihydroflavones. It has anti-ischemic effects and anti-hemagglutination effects. After the compatibility of various drugs, JZGXP has the effect of promoting blood circulation and removing blood stasis and also is commonly used in the treatment of CHD.

Mass spectrometry-based metabolomics is one of the effective tools for studying the therapeutic mechanisms of traditional Chinese medicines and traditional Chinese medicine formulations. ${ }^{15-17}$ After 28 days of treatment, the results of clinical biochemical indicators showed that compared with the model group, the serum levels of TG and CHO in rats treated with JZGXP decreased, and the serum HDL-C content increased. JZGXP improved the blood lipid level of rats. Results of the cardiac index showed that compared with the model group, the cardiac index of the rats in the JZGXP injection group had an increasing trend, indicating that the JZGXP improved the large cardiac index of the rats. Electrocardiogram results showed that after treatment with JZGXP, the myocardial ischemia status of the rats disappeared, which improved the myocardial ischemia in rats. Results of HE staining of coronary and myocardial samples showed that compared with the model group the deposition of lipid cells in rats treated with JZGXP was significantly reduced, the deposition of calcium salts disappeared, and the degree of myocardial fibrosis was significantly improved, indicating that JZGXP improved the degree of

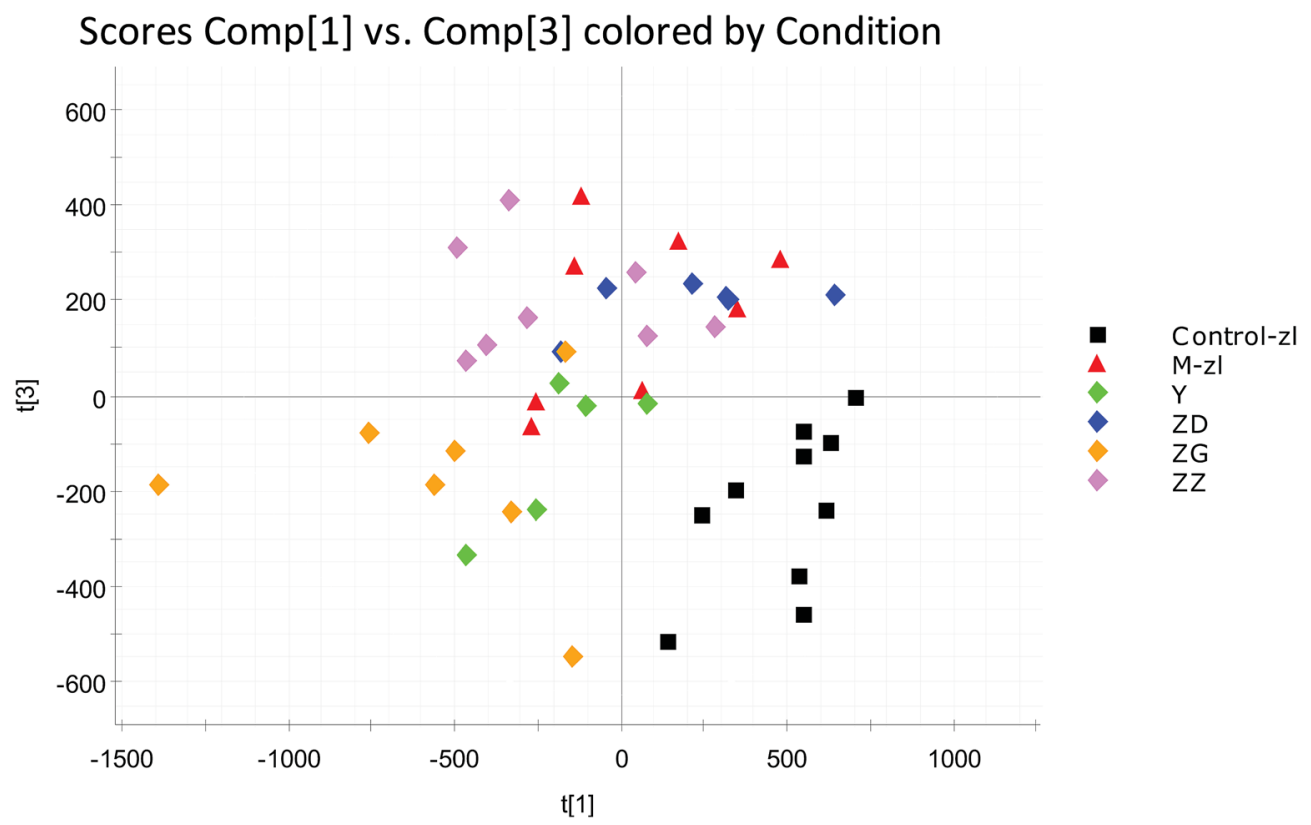

Fig. 8 Score map of urine metabolic profiles of rats after 28 days of administration (OPLS-DA). Control-zl: control group; M-zl: model group; Y: positive group; ZD: the low dosage JZGXP group; ZG: the high dosage JZGXP group; ZZ: the middle dosage JZGXP group. 

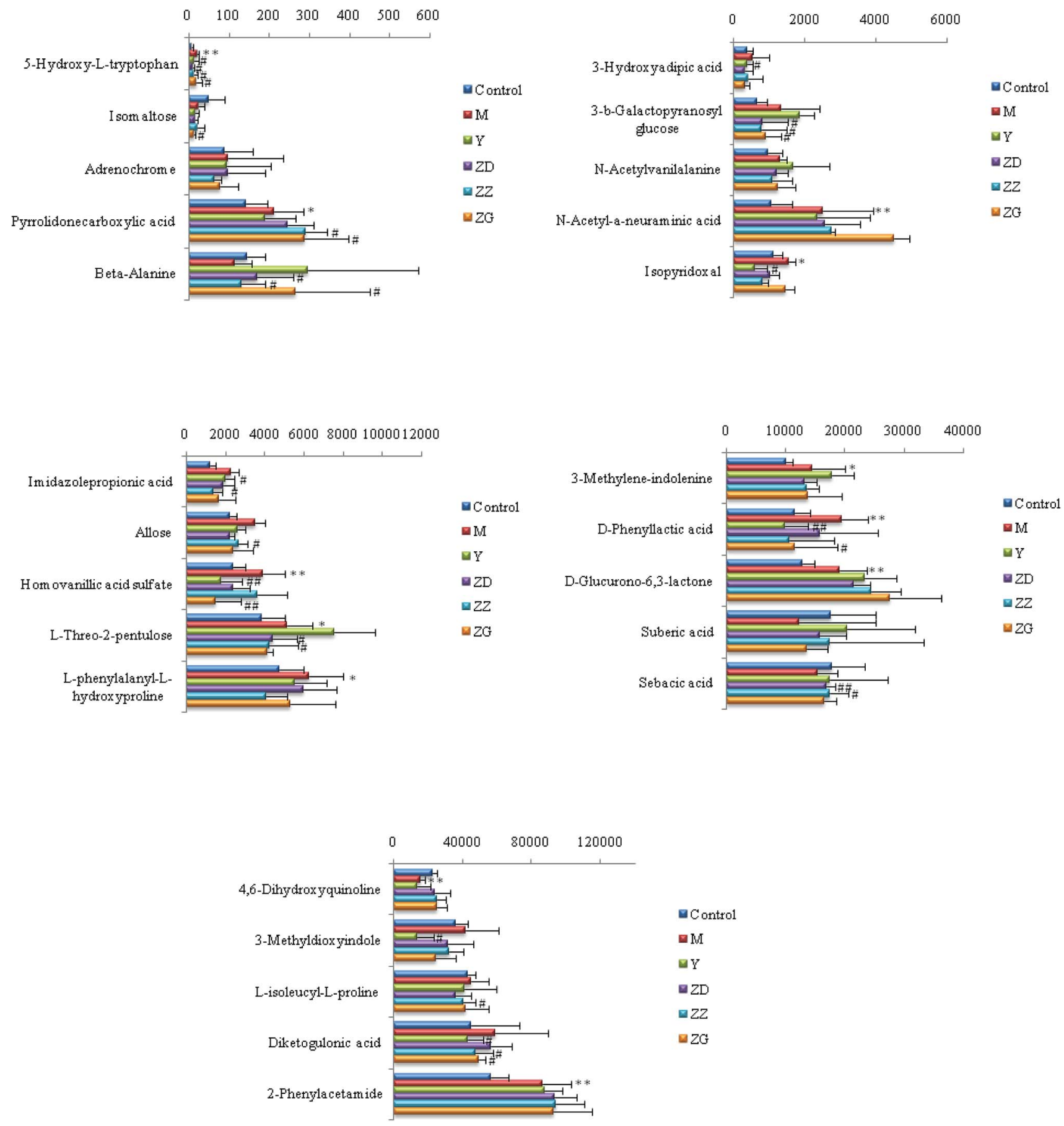

Fig. 9 Content changes of CHD model rat urine biomarkers after the oral administration of JZGXP. Control: control group; M: model group; Y: positive group; ZD: the low dosage JZGXP group; ZZ: the middle dosage JZGXP group; ZG: the high dosage JZGXP group (compared to control group: $* P<0.05,{ }^{*} P<<0.01$; compared to model group: $\# P<0.05$, $\# P<0.01$ ).

atherosclerosis. As described above, JZGXP can improve CHD and have a certain degree of therapeutic effects on CHD.

Metabolomics plays an important role in exploring metabolic disorder metabolism-related diseases, ${ }^{18-29}$ biomarkers identification ${ }^{30-38}$ and response to treatment. ${ }^{39-46}$ In this study, differences in biomarkers in urine between the model group and the blank group were successfully analyzed and 25 biomarkers were ultimately associated with the CHD model.
Further research found that these 25 biomarkers involved 9 metabolic pathways in the body, which were summarized as amino acid metabolism, carbohydrate metabolism, nucleotide metabolism, vitamin and coenzyme metabolism and other amino acids metabolism and showed that CHD disturbs these five metabolisms in the body. JZGXP can recall 21 biomarkers in the urine of model rats. The metabolism of these biomarkers was mainly the same as above. From the micro level, it is 
indicated that the JZGXP plays a therapeutic role in CHD by recalling biomarkers in the metabolic network map.

CHD can cause an imbalance of amino acid metabolism, including $\beta$-alanine metabolism disorders and tyrosine metabolism disorders. Biomarkers directly related to $\beta$-alanine metabolism and tyrosine metabolism are $\beta$-alanine and high vanillic acid sulfate. After treatment with JZGXP, the contents of $\beta$-alanine and high vanillic acid sulfate in the body can be adjusted back to that of the normal group. This indicates that JZGXP can exert therapeutic effects by normalizing $\beta$-alanine metabolism and tyrosine metabolism.

\section{Conclusions}

We applied a metabolomics strategy to reveal the biomarkers and related metabolic pathway networks of CHD models and found potential biomarkers and therapeutic mechanisms of JZGXP in the treatment of CHD. JZGXP can play a certain therapeutic role in rats with $\mathrm{CHD}$, mainly through regulating the body's amino acid metabolic pathway, carbohydrate metabolic pathway, nucleotide metabolic pathway, vitamin and coenzyme metabolic pathways and other amino acid metabolic pathways. It can effectively improve the pathological state of CHD. The main targets are $\beta$-alanine metabolism and tyrosine metabolism, i.e., amino acids metabolism. In short, we used theoretical guidance from traditional Chinese medicine to establish a rat model of CHD and evaluated it using clinical biochemical indicators and histopathological diagnosis. From the perspective of metabolomics, the potential biomarkers and therapeutic mechanisms of JZGXP for the treatment of CHD were elucidated. Our research indicates that metabolomics is an effective method for discovering and identifying biomarkers related to diseases or syndromes such as CHD and discovering therapeutic mechanisms for traditional Chinese medicine formulations.

\section{Conflicts of interest}

There are no conflicts to declare.

\section{Acknowledgements}

This work was supported by grants from the Key Program of the Natural Science Foundation of State (Grant No. 81830110, 81430093, 81373930, 81673586, 81703685, 81302905, 81503386), the National Key Subject of Drug Innovation (Grant No. 2015ZX09101043-005, 2015ZX09101043-011), Major Projects of Application Technology Research and Development Plan in Heilongjiang Province (GX16C003), the TCM State Administration Subject of Public Welfare (Grant No. 2015468004), the University Nursing Program for Young Scholars with Creative Talents in Heilongjiang Province (UNPYSCT-2015118, UNPYSCT-2016213, UNPYSCT-2016212), the Young Talent Lift Engineering Project of China Association of Traditional Chinese Medicine (QNRC2-B06), and the Outstanding Talents Foundation of Heilongjiang University of Chinese Medicine (2018jc01).

\section{References}

1 M. J. Mathews, E. H. Mathews and G. E. Mathews, The integrated effect of moderate exercise on coronary heart disease, Cardiovasc. J. Afr., 2017, 28(2), 125-133.

$2 \mathrm{M}$. Orho-Melander, Genetics of coronary heart disease: towards causal mechanisms, novel drug targets and more personalized prevention, J. Intern. Med., 2015, 278(5), 433446.

3 Z. Pang, W. Zhao and Z. Yao, Cardioprotective Effects of Nicorandil on Coronary Heart Disease Patients Undergoing Elective Percutaneous Coronary Intervention, Med. Sci. Monit., 2017, 23, 2924-2930.

4 Q. Feng, Z. Liu, S. Zhong, R. Li, H. Xia, Z. Jie, B. Wen, X. Chen, W. Yan, Y. Fan, Z. Guo, N. Meng, J. Chen, X. Yu, Z. Zhang, K. Kristiansen, J. Wang, X. Xu, K. He and G. Li, Integrated metabolomics and metagenomics analysis of plasma and urine identified microbial metabolites associated with coronary heart disease, Sci. Rep., 2016, 6, 22525.

5 M. Zhao, I. Vaartjes, I. Graham, D. Grobbee, W. Spiering, K. Klipstein-Grobusch, M. Woodward and S. A. Peters, Sex differences in risk factor management of coronary heart disease across three regions, Heart, 2017, 103(20), 15871594.

6 Z. Li, T. Liu, J. Han, T. Li, Q. Zhu and A. Wang, Confrontation as a Mediator between Sense of Coherence and Selfmanagement Behaviors among Elderly Patients with Coronary Heart Disease in North China, Asian Nurs. Res. (Korean Soc. Nurs. Sci.), 2017, 11(3), 201-206.

7 Y. H. Li, X. Huang, Y. Wang, R. Fan, H. M. Zhang, P. Ren, Y. Chen, H. H. Zhou, Z. Q. Liu, Y. Z. Liang and H. M. Lu, Pharmacokinetic comparison of the vasorelaxant compound ferulic acid following the administration of Guanxin II to healthy volunteers and patients with angina pectoris, Exp. Ther. Med., 2013, 6(5), 1283-1289.

8 X. Qiao, J. Han, M. Xu, Z. C. Zhang, M. Ye, G. Ye and D. A. Guo, Metabolic profiling of GuanXin II prescription based on metabolic fingerprinting and chemical analysis, $J$. Pharm. Biomed. Anal., 2011, 54(4), 789-798.

9 Y. Ren, Z. Q. Chen, M. Z. Zhang, L. H. Guo and D. Y. He, [Cluster Analysis of Medication Laws for Treating Coronary Heart Disease by Distinguished Veteran Doctors of Traditional Chinese Medicine], Zhongguo Zhongxiyi Jiehe Zazhi, 2016, 36(4), 411-414.

10 A. Zhang, H. Sun, G. Yan, P. Wang and X. Wang, Mass spectrometry-based metabolomics: applications to biomarker and metabolic pathway research, Biomed. Chromatogr., 2016, 30(1), 7-12.

11 F. X. Ma, P. F. Xue, Y. Y. Wang, Y. N. Wang and S. Y. Xue, Research progress of serum pharmacochemistry of traditional Chinese medicine, Zhongguo Zhongyao Zazhi, 2017, 42(7), 1265-1270.

12 F. R. Zhao, J. X. Lu, M. Jia, Y. L. Yin, H. T. Qi, M. L. Zhu, L. J. Ma, L. L. Qiu, G. M. Wan and G. R. Wan, Effects and 
mechanism of Xin Mai Jia in a rabbit model of atherosclerosis, Exp. Ther. Med., 2015, 10(5), 1627-1634.

13 A. H. Zhang, H. Sun, S. Qiu and X. J. Wang, Recent highlights of metabolomics in chinese medicine syndrome research, $J$. Evidence-Based Complementary Altern. Med., 2013, 2013, 402159.

14 T. Zhang, A. Zhang, S. Qiu, S. Yang and X. Wang, Current Trends and Innovations in Bioanalytical Techniques of Metabolomics, Crit. Rev. Anal. Chem., 2016, 46(4), 342-351.

15 S. Qiu, A. Zhang, T. Zhang, H. Sun, Y. Guan, G. Yan and X. Wang, Dissect new mechanistic insights for geniposide efficacy on the hepatoprotection using multiomics approach, Oncotarget, 2017, 8(65), 108760-108770.

16 X. Li, A. Zhang, H. Sun, Z. Liu, T. Zhang, S. Qiu, L. Liu and $\mathrm{X}$. Wang, Metabolic characterization and pathway analysis of berberine protects against prostate cancer, Oncotarget, 2017, 8(39), 65022-65041.

17 T. Zhang, A. Zhang, S. Qiu, H. Sun, Y. Han, Y. Guan and X. Wang, High-throughput metabolomics approach reveals new mechanistic insights for drug response of phenotypes of geniposide towards alcohol-induced liver injury by using liquid chromatography coupled to high resolution mass spectrometry, Mol. BioSyst., 2016, 13(1), 73-82.

18 A. H. Zhang, J. B. Yu, H. Sun, et al., Identifying qualitymarkers from Shengmai San protects against transgenic mouse model of Alzheimer's disease using chinmedomics approach, Phytomedicine, 2018, (18), 30104-301011.

19 (a) H. Sun, A. Zhang, Q. Song, et al., Functional metabolomics discover pentose and glucuronate interconversion pathways as promising targets for Yang Huang syndrome treatment with Yinchenhao Tang, RSC Adv., 2018, 8(64), 36831-36839; (b) A. Zhang, H. Sun, G. Yan, et al., Chinmedomics: A Powerful Approach Integrating Metabolomics with Serum Pharmacochemistry to Evaluate the Efficacy of Traditional Chinese Medicine. Engineering, 2018, DOI: 10.1016/j.eng.2018.11.008.

20 A. Zhang, H. Sun, G. Yan, et al., Metabolomics Analysis of Marker Metabolites for Patients with Pancreatic Cancer, Medicinal Chemistry, 2014, 4, 506-510.

21 X. Wang, H. Lv, A. Zhang, et al., Metabolite profiling and pathway analysis of acute hepatitis rats by UPLC-ESI MS combined with pattern recognition methods, Liver Int., 2014, 34(5), 759-770.

22 A. Zhang, H. Sun, H. Xu, et al. Cell metabolomics, OMICS, 2013, 17(10), 495-501.

23 H. Sun, A. H. Zhang, S. B. Liu, et al., Cell metabolomics identify regulatory pathways and targets of magnoline against prostate cancer, J. Chromatogr. B: Anal. Technol. Biomed. Life Sci., 2018, 1102-1103, 143-151.

24 A. Zhang, H. Sun and X. Wang, Urinary metabolic profiling of rat models revealed protective function of scoparone against alcohol induced hepatotoxicity, Sci. Rep., 2014, 4, 6768.

25 X. Liu, A. Zhang, H. Fang, et al., Serum metabolomics strategy for understanding the therapeutic effects of YinChen-Hao-Tang against Yanghuang syndrome, RSC Adv., 2018, 8(14), 7403-7413.
26 A. Zhang, H. Sun, G. Yan, et al., Metabolomics study of type 2 diabetes using ultra-performance LC-ESI/quadrupole-TOF high-definition MS coupled with pattern recognition methods, J. Physiol. Biochem., 2014, 70(1), 117-128.

27 Q. Song, A. Zhang, G. Yan, et al., Technological advances in current metabolomics and its application in tradition Chinese medicine, RSC Adv., 2017, 7(84), 53516-53524.

28 A. Zhang, H. Sun, W. Sun, et al., Metabolomics and Proteomics Annotate Therapeutic Mechanisms of Geniposide[M]// Chinmedomics, Academic Press, 2015, pp. 157-173.

29 Q. Zhao, A. Zhang, W. Zong, et al., Chemometrics strategy coupled with high resolution mass spectrometry for analyzing and interpreting comprehensive metabolomic characterization of hyperlipemia, $R S C A d v ., 2016,6(113)$, 112534-112543.

30 X. Wang, Y. Han, A. Zhang, et al., Metabolic profiling provides a system for the understanding of Alzheimer's disease in rats post-treatment with Kaixin San[M]//Chinmedomics, Academic Press, 2015, pp. 347-362.

31 A. Zhang, H. Wang, H. Sun, et al., Metabolomics strategy reveals therapeutical assessment of limonin on nonbacterial prostatitis, Food Funct., 2015, 6(11), 3540-3549.

32 (a) J. L. Ren, A. H. Zhang, L. Kong, et al., Advances in mass spectrometry-based metabolomics for investigation of metabolites, RSC Adv., 2018, 8(40), 22335-22350; (b) A. Zhang, H. Sun and X. Wang, Mass spectrometry-driven drug discovery for development of herbal medicine, Mass Spectrom. Rev., 2018, 37(3), 307-320.

33 H. Sun, M. Wang, A. Zhang, et al., UPLC-Q-TOF-HDMS Analysis of Constituents in the Root of Two Kinds of Aconitum Using a Metabolomics Approach, Phytochem. Anal., 2013, 24(3), 263-276.

34 F. Wu, H. Sun, W. Wei, et al., Rapid and global detection and characterization of the constituents in ShengMai San by ultra-performance liquid chromatography-high-definition mass spectrometry, J. Sep. Sci., 2011, 34(22), 3194-3199.

35 X. Wang, A. Zhang, H. Sun, et al., Discovery and development of innovative drug from traditional medicine by integrated chinmedomics strategies in the post-genomic era, $\operatorname{TrAC}$, Trends Anal. Chem., 2016, 76, 86-94.

36 A. Zhang, H. Sun, S. Qiu, et al., Advancing drug discovery and development from active constituents of yinchenhao tang, a famous traditional chinese medicine formula, J. EvidenceBased Complementary Altern. Med., 2013, 2013.

37 X. Wang, Q. Wang, A. Zhang, et al., Metabolomics study of intervention effects of Wen-Xin-Formula using ultra highperformance liquid chromatography/mass spectrometry coupled with pattern recognition approach, J. Pharm. Biomed. Anal., 2013, 74, 22-30.

38 W. Dong, P. Wang, X. Meng, et al., Ultra-performance Liquid Chromatography-High-definition Mass Spectrometry Analysis of Constituents in the Root of Radix Stemonae and those Absorbed in Blood after Oral Administration of the Extract of the Crude Drug, Phytochem. Anal., 2012, 23(6), 657-667.

39 X. J. Wang, J. L. Ren, A. H. Zhang, et al., Novel applications of mass spectrometry-based metabolomics in herbal 
medicines and its active ingredients: Current evidence, Mass Spectrom. Rev., 2019, 9999, 1-23.

40 H. Wang, G. Yan, A. Zhang, et al., Rapid discovery and global characterization of chemical constituents and rats' metabolites of Phellodendri amurensis cortex by ultraperformance liquid chromatography-electrospray ionization/quadrupole-time-of-flight mass spectrometry coupled with pattern recognition approach, Analyst, 2013, 138(11), 3303-3312.

41 C. Liu, A. Zhang, G. L. Yan, et al., High-throughput ultra high performance liquid chromatography coupled to quadrupole time-of-flight mass spectrometry method for the rapid analysis and characterization of multiple constituents of Radix Polygalae, J. Sep. Sci., 2017, 40(3), 663-670.

42 A. Zhang, H. Sun and X. Wang, Emerging role and recent applications of metabolomics biomarkers in obesity diseases research, RSC Adv., 2017, 7, 14966-14973.

43 X. Wang, Q. Wang, A. Zhang, et al., Metabolomics study of intervention effects of Wen-Xin-Formula using ultra high- performance liquid chromatography/mass spectrometry coupled with pattern recognition approach, J. Pharm. Biomed. Anal., 2013, 74, 22-30.

44 H. Sun, H. Zhang, A. Zhang, et al., Network pharmacology combined with functional metabolomics discover bile acid metabolism as a promising target for mirabilite against colorectal cancer, RSC Adv., 2018, 8(53), 30061-30070.

45 H. Zhang, A. Zhang, X. Zhou, et al., High-throughput lipidomics reveal mirabilite regulating lipid metabolism as anticancer therapeutics, RSC Adv., 2018, 8(62), 35600-35610.

46 H. Zhang, A. Zhang, J. Miao, et al., Targeting regulation of tryptophan metabolism for colorectal cancer therapy: a systematic review, RSC Adv., 2019, 9(6), 3072-3080.

47 X. N. Li, A. Zhang, H. Sun, Y. Song, D. Zou and X. Wang, Rapid discovery of absorbed constituents and metabolites in rat plasma after the oral administration of $\mathrm{Zi}$ Shen Wan using high-throughput UHPLC-MS with a multivariate analysis approach, J. Sep. Sci., 2016, 39(24), 4700-4711. 